\title{
Research on the Reconstruction of Shaanxi's New Spatial Pattern of Economic Development from the Perspective of Economic Geography
}

\author{
Xuqi Zhang \\ Xi'an International University, Xi'an, Shaanxi, China
}

Keywords: Economic Geography; Economic Density; Guanzhong Urban Agglomeration.

\begin{abstract}
Reconstructing Shaanxi's economic geography and giving full play to Shaanxi's geographical advantages in Guanzhong area is a new way to catch up with and surpass Shaanxi's economy. Based on the theory of economic geography, this paper studies the driving effect of economic geography reconstruction on Shaanxi's economic growth from three dimensions: distance, density and segmentation. Based on the economic characteristics of Guanzhong urban agglomeration in Shaanxi Province, this paper summarizes that industrial agglomeration effect, migration, specialization and trade are the main driving forces for reconstructing Shaanxi's economic geography, and puts forward a new pattern of economic development space for increasing economic output density and economic increment, aiming at forming Shaanxi's characteristic economic growth pole and promoting Shaanxi's comprehensive competition power.
\end{abstract}

\section{Introduction}

Shaanxi Guanzhong Urban Agglomeration undertakes the historical mission of leading the highspeed and sustainable development of Shaanxi's economy. With the approval of Shaanxi Free Trade Zone and the implementation of the "One Belt and One Road" strategy and other new situations and opportunities, the reconstruction of Shaanxi's new economic and geographic pattern will play an active role in promoting the rapid economic growth of Shaanxi and the goal of building an international metropolis.The Background of College Students' Entrepreneurship

\section{A Survey of Economic Geography of Guanzhong Urban Agglomeration in Shaanxi Province}

In 1760, Russian scientist Romonosov first put forward the name "economic geography", pointing out that the study of national economy must be combined with geographical conditions. Spatial pattern is one aspect of economic development. The development of regional economy should conform to the natural law of economic geography. It is long-term and feasible to plan and design the spatial pattern of Shaanxi's economic development according to the geographical characteristics of regional economy and the experience of international economic geography. 


\subsection{Scale Analysis of Guanzhong Urban Agglomeration in Shaanxi Province}

The urban agglomeration in Guanzhong area of Shaanxi Province refers to the continuous urban belt with Xi'an-Xianyang metropolitan area as the center, east to Tongguan West to Baoji City and north to Tongchuan City, including 8 cities, 1 agricultural demonstration area, 5 prefecture-level cities, 3 county-level cities and 404 small towns. Land area of 55,500 square kilometers, accounting for $26.9 \%$ of the province's area, population of 23,403,400, accounting for $62.7 \%$ of the province's population, per capita GDP of 56,988 yuan, which is about $33 \%$ higher than the average of the province. (Table 1) Guanzhong Urban Agglomeration is the largest urban agglomeration in Northwest China, and also the gateway and link of Northwest China's external relations. It has a wide range of radiation. Its development level directly restricts the development level of Shaanxi's economy, and also affects the development of the whole western region.

Table 1 Size Structure of Guanzhong Urban Agglomeration

\begin{tabular}{ccccc}
\hline City & $\begin{array}{c}\text { District and } \\
\text { county }\end{array}$ & $\begin{array}{c}\text { Population } \\
\text { (thousand) }\end{array}$ & GDP(Billion yuan) & $\begin{array}{c}\text { Land area } \\
\left(\mathbf{K M}^{\mathbf{2}}\right)\end{array}$ \\
\hline Xi'an & 13 & 8588.1 & 488.4 & 10108 \\
Xianyang & 13 & 4942.0 & 186.0 & 10025 \\
Baoji & 12 & 3744.6 & 154.51 & 18172 \\
WeinanTongc & 11 & 5331.7 & 134.9 & 13046 \\
huan & 4 & 842.8 & 3.29 & 3882 \\
Yangling & 1 & 202.4 & 8.47 & 94 \\
\hline
\end{tabular}

Data Source: Based on the Statistical Yearbook of Shaanxi Province in 2017

\subsection{Industrial Agglomeration Analysis of Guanzhong Urban Agglomeration}

Agricultural agglomeration area: Guanzhong is the main grain producing area in China. Grain production accounts for more than $50 \%$ of the whole province, and its grain yield per mu is $27.5 \%$ higher than that of the whole province, $56.5 \%$ of lean meat type pig production base and $77.3 \%$ of apple base. The economic scale of pomegranate in Lintong, kiwifruit in BaojiMei County, Boer goat in BaojiLinyou Tour, Xingping dairy cow and beef cattle in Xianyang are taking shape.

Industrial agglomeration: Weinan and Baoji non-ferrous

metal mineral resources; Tongchuan and Hancheng coal resources, Shaanxi is an important energy and mineral base. The eastern suburbs of Xi'an have formed clusters of weaponry industry, electric power equipment, instruments and machinery processing industry; Yanliang District around Xi'an has formed aerospace industry clusters of aircraft design, R\&D, production and flight test; Xianyang textile industry cluster, Xi'an western suburbs high-tech industry cluster, etc. The division of labor in Guanzhong city is different, and their functions are mutually interdependent. Supplementary urban pattern.

\section{The Spatial Distribution Characteristics of Guanzhong Urban Agglomeration}

\subsection{Small-scale urban agglomeration}

Compared with Yangtze River Delta, Pearl River Delta and Beijing-Tianjin-Hebei urban agglomeration, Guanzhong urban agglomeration has fewer population and smaller scale. ( Table 2) 
Table 2 Development of Ten Urban Agglomerations in China in 2015

\begin{tabular}{cccc} 
Urban Agglomeration & $\begin{array}{c}\text { population } \\
\text { (thousand) }\end{array}$ & GDP (Billion) & $\begin{array}{c}\text { Urbanization } \\
\text { rate \% }\end{array}$ \\
\hline BeijingTianjin Hebei & 106140 & 5141.9 & 67.5 \\
Changjiang Delta & 115800 & 9725.2 & 56.3 \\
Pearl River Delta & 55410 & 5380.2 & 74.5 \\
Middle Yangtze & 112430 & 4121.3 & 57.2 \\
Chengdu Chongqing & 83760 & 2522.4 & 51.7 \\
WestcoastofTaiwan Strait & 88350 & 2908.9 & 48.8 \\
Shandong Peninsula & 45560 & 3234.6 & 58.0 \\
South Central Liaoning & 37000 & 2444.6 & 60.9 \\
Central Plains & 41540 & 1744.9 & 47.9 \\
Guanzhong & 23500 & 772.2 & 51.6 \\
Guanzhong ranking & $\mathbf{1 0}$ & $\mathbf{1 0}$ & $\mathbf{8}$ \\
\hline
\end{tabular}

Data Source: References 5

\subsection{Lack of Large Cities}

The scale structure of Guanzhong urban agglomeration is imperfect. There is only one super-city in Xi'an, and there is no super-city in Guanzhong urban agglomeration. Baoji is the only big city in Guanzhong urban agglomeration, while Xianyang, Weinan, Tongchuan and Hancheng, Xingping and Huayin are the medium-sized cities, which account for a large proportion. The lack of the transition and inheritance of large cities weakens the radiation driving role of Xi'an as a mega-city.

\subsection{Unbalanced Urban Development}

Table 3 Industrial Location Quotient and Specialization of Guanzhong City

\begin{tabular}{|c|c|c|c|c|c|}
\hline Industrial Name & Xi'an & Tongchuan & Baoji & Xianyang & Weinan \\
\hline Agroforestry & 0.509 & 0.580 & $1.372 \nless$ & $1.292 \nless$ & $1.990 \nmid$ \\
\hline $\begin{array}{l}\text { Extractive industry } \\
\text { manufacturing industry }\end{array}$ & 0.037 & $7.236 \star$ & 0.444 & 0.313 & $3.246 \star$ \\
\hline $\begin{array}{l}\text { Electric coal supply } \\
\text { Construction business }\end{array}$ & $1.065 \star$ & 0.784 & $1.208 \star$ & $1.013 \star$ & 0.635 \\
\hline $\begin{array}{l}\text { Logistics } \\
\text { trade }\end{array}$ & 0.696 & 0.713 & 0.815 & $1.295 \star$ & $1.927 \star$ \\
\hline $\begin{array}{l}\text { Financial insurance } \\
\text { Socialservices }\end{array}$ & $0.985 \star$ & $0.883 \star$ & 0.840 & $1.564 \star$ & 0.610 \\
\hline Health ports & $1.234 \star$ & 0.760 & $1.458 \star$ & 0.467 & 0.498 \\
\hline \multirow[t]{6}{*}{ Education Culture } & $1.116 \star$ & 0.547 & $1.037 \star$ & 0.895 & 0.869 \\
\hline & $1.126 \star$ & 0.701 & 0.903 & $1.697 \star$ & $1.152 \star$ \\
\hline & $1.415 \star$ & 0.578 & 0.641 & 0.529 & 0.742 \\
\hline & $1.460 \star x$ & 0.481 & 0.681 & 0.587 & 0.552 \\
\hline & 0.943 & 0.621 & $1.019 \nless$ & $1.197 \nmid$ & $1.071 \nless$ \\
\hline & 0.897 & 0.589 & 0.890 & 1.228 ׳ & $1.328 \star$ \\
\hline
\end{tabular}

Data Source: Based on the Statistical Yearbook of Shaanxi Province in 2017

Location quotient, also known as specialization rate, is a quantitative tool for the analysis of efficiency and efficiency of industries, which can analyze the situation of regional superior industries. Generally speaking, if the location quotient of the industry is greater than 1.5, then the industry has 
obvious comparative advantages in the local area. The following table 3 shows that the development of the main urban agglomerations in Guanzhong is extremely unbalanced, the degree of specialization is low, and the correlation between cities is low.

\section{Analysis of Economic and Geographical Indicators of Guanzhong Urban Agglomeration}

\subsection{Uneven Density of Guanzhong Urban Agglomeration in Shaanxi Province}

(1) The uneven density of population and economic output

The theory of economic geography points out that "density refers to the total economic amount per unit of land, and it is a vital feature of the local development level". The population of five cities and one district of Guanzhong urban agglomeration in Shaanxi Province accounts for $62.78 \%$ of the total population of Shaanxi Province, and the total GDP value is 77.211 billion yuan, accounting for $61.72 \%$ of the total GDP of Shaanxi Province. The area accounts for $26.9 \%$ of the province's total area, gathering $80 \%$ of the scientific and technological strength of the province and $98 \%$ of the total import and export value. There are 52 colleges and Universities under the jurisdiction, 6"211 Engineering Colleges "In addition private undergraduate colleges, the number is more than 70, which is Second only to Beijing. GDP output per square kilometer is 3-4 times the average of the province. On average, there is one city per 6937.5 kilometers and one town per 137.4 square kilometers. On average, there is only one city in the northwest of China, and only one town in 2176 square kilometers. Because of the agglomeration effect of the central city Xi'an, the development of other cities in the urban agglomeration is unbalanced.

Table 4 Percentage of Industrial Output and Population Distribution in Guanzhong Cities

\begin{tabular}{ccc}
\hline City & Proportion of gross industrial output value $(\%)$ & Population ratio (\%) \\
\hline Xi'an & 45.3 & 32.7 \\
Tongchuan & 3.3 & 3.8 \\
Baoji & 18.6 & 16.6 \\
Xianyang & 17.9 & 22.1 \\
Weinan & 14.0 & 24.2 \\
Yanglin & 0.9 & 0.6 \\
\hline
\end{tabular}

Data Source: Statistical Yearbook of Shaanxi Province 2017

(2) High industrial convergence of Urban Agglomerations

The convergence of specialized industries in Guanzhong urban agglomeration will inevitably lead to excessive competition and insufficient complementarity among cities. This kind of industrial layout and convergence deviates from the law of regional distribution of labor and objective requirements, affects the rationality of functional division of labor among cities, and easily leads to competition between cities and enterprises for limited resources. As a result of urban agglomeration, competition exists among cities (Table 3 Annotations $\star$ )

\subsection{The Distance of Guanzhong Urban Agglomeration in Shaanxi Province is too Long}

Economic geography points out that "distance refers to the difficulty of goods, services, services, capital, information and ideas traversing space. It is a vital feature of the national development level." The cities of Xi'an, Xianyang, Weinan and Baoji in Guanzhong City Cluster of Shaanxi Province are located on the Longhai Line, which belongs to the typical zonal distribution of highspeed gathering in the East-West direction. In addition, the Longhai Line, Xibao Expressway and Xilin Expressway run through these cities, making transportation very convenient. However, most of the small and medium-sized cities are located in hilly areas and mountainous areas, far from the 
Longhai Line, such as Tongchuan, Hancheng and Xingping. Villages and towns also radiate to the surrounding mountains and hills with the city as the center. Because of the terrain and roads, the straight line distance between townships in different urban jurisdictions is not far, but it is inconvenient to achieve. The distance between villages and towns is shown in Table 5 (centering on Xi'an, the distribution is decreasing and diverging around it).

Table 5 Distribution Distance of Cities and Towns in Guanzhong Urban Agglomeration

\begin{tabular}{cccc}
\hline Prefecture-level City & County-level City & $\begin{array}{c}\text { Number of County } \\
\text { Towns }\end{array}$ & $\begin{array}{c}\text { Average Distance } \\
(\mathbf{k m})\end{array}$ \\
\hline Xianyang & Xingping & 7 & 50 \\
Weinan & Yangling & 13 & $50 \sim 100$ \\
Tongchuan & Huayin & 13 & $100 \sim 150$ \\
Baoji & Hancheng & 5 & $150 \sim 200$ \\
\hline
\end{tabular}

Data Source: References 5

\subsection{The Urban Agglomeration of Guanzhong in Shaanxi Province has Obvious Degree of Segmentation}

Economic geography points out that "segmentation refers to barriers to economic interaction caused by currency, tariff and linguistic differences. It is the most important feature of development at the international level."

Located at the center of the whole territory of China, Guanzhong Urban Agglomeration has a very important strategic position. The state has increased its investment in China's defense industry for the need of national defense, and all of them are capital-intensive and technology-intensive industries, belonging to the type of heavy industrialization and nationalization, which deviates from the comparative advantages and dynamic advantages of Guanzhong region itself. Potential, ignoring the resources endowment of the region, weakened the links and cooperation with the surrounding cities, hindered the progress of Shaanxi's marketization, and created a division with other cities and regions.

Secondly, Shaanxi Guanzhong Aerospace and Aerospace Enterprises, Military Industrial Enterprises and Heavy Industries are in the leading position. These industries belong to nationalization, monopoly production and operation under the state-owned system, and state protection. They have high market barriers for foreign investment, inter-city cooperation and the entry of private enterprises.

\section{Measures for Shaanxi's Economic and Geographical Reconstruction}

Economic geography points out that "increasing density, shortening distance and breaking up division are the basic ways to promote economic geography change". Shaanxi Guanzhong region has many advantages over southern and Northern Shaanxi. Reconstructing Shaanxi's economic geography, giving full play to the driving potential of Guanzhong urban agglomeration and realizing the leap-forward development of the whole province's economy have become the basic strategy of Shaanxi's regional social and economic development.

\subsection{Shortening the Time Distance between Cities}

We will improve the transportation network system of Guanzhong urban agglomerations with Xi'an as the center and coordinated development of various modes of transport. Establishing a multilevel and network-based sub-development framework in the region will enable urban groups and multiple development axes to present an organic and distinct network-based configuration. Through 
the metre-shaped transportation network composed of Railways and trunk highways, the regional spatial connection is strengthened, and the spatial structure of urban agglomerations is based on it.

The first is to focus on the construction of the Longhai Line, the expansion of Xibao, Xitong Expressway-based east-west traffic corridor. We will improve the trunk lines of Baocheng, Houxi, Xiyan, Xiantong, Baozhong railways, 108, 210, 211, 310 and 312, as well as the expressways and provincial highways of Xitong, and form a highway network of "three horizontal, four vertical and five radiation". Strengthen the construction of expressways between Xi'an, Baoji, Weinan, Tongchuan and Yangling. Highway routes constitute the highway loop of Guanzhong urban agglomeration and form an inter-city trunk line network in the core area.

The second is to speed up the construction of high-speed railways, form a network of rapid transportation circles with Xi'an as the center, reaching the surrounding provincial capital cities in 23 hours and reaching the Bohai Sea, Yangtze River Delta and Pearl River Delta in 5-6 hours, increase the accessibility and convenience between provincial cities, shorten the economic distance within and among regions, and form a public transportation network as the basis. Spatial structure of urban agglomeration industry promotes the economic development of surrounding counties

Third, actively expand Xi'an's international flights to Southeast Asia, Oceania, Europe and the United States, and increase the number of countries The density of internal flights has formed an aviation network centered on Xianyang Airport in Xi'an, connecting important cities at home and abroad, making civil air transportation an important part of Guanzhong's transportation system.

With the improvement of the transportation network of Guanzhong urban agglomeration, the regional structure of Guanzhong urban agglomeration will be guided and promoted to change, so that economic Unicom will gradually spread to the whole urban agglomeration through the functional and hierarchical division of labor among cities, and the economic diffusion effect and penetration effect of Guanzhong urban agglomeration will be further developed.

\subsection{Increasing the Density of Urban Economic Output}

(1) Industrial agglomeration to increase economic output density

According to the superior industries in different regions, we should try to create and guide industrial clusters and build up regional competitive advantages: Xi'an should focus on developing information industry, aerospace industry, biology and new medicine industry clusters, Baoji should focus on developing new materials, advanced manufacturing and Mechatronics Industry clusters, etc. South relies on economic and Technological Development zones, focusing on the development of industrial clusters in chemical, non-ferrous metals and food processing industries; Tongchuan should promote the level of development of energy, building materials and non-ferrous industries.

High-tech industrial parks are helpful to form the industrial intensive zone of Guanzhong urban agglomeration. In recent years, the national high-tech industrial parks in Xianyang, Weinan, Yulin, Ankang, Hanzhong and Tongchuan provincial high-tech zones have been gradually established, attracting population and industrial agglomeration, promoting urbanization, forming industryintensive zones, and promoting unit-level improvement. Accumulated economic output.

(2) Spatial distribution of three-tier industries, forming dense economic circles

Create a circle-level economic and geographical pattern around Xi'an:

The first level is the manufacturing industrial agglomeration area with Xi'an as the core. It mainly focuses on the labor-intensive manufacturing industry and capital-technology-intensive manufacturing industry, such as tobacco processing, textile, furniture manufacturing, cultural, educational and sporting goods and other labor-intensive industries and capital technology, such as general equipment, special equipment, electrical machinery, instruments and meters. Intensive industries; 
The second level is a resource-intensive manufacturing and productive labor-intensive manufacturing agglomeration area centered around Baoji, Xianyang and Weinan, such as petroleum processing, ferrous metal smelting, non-ferrous metal smelting, food manufacturing, food processing, wood processing, general equipment, etc.

The third level is a transitional industrial agglomeration area composed of Tongchuan. Apart from resource-intensive manufacturing, Tongchuan has gradually become a agglomeration area for food and beverage industries.

\subsection{Breaking the Dividing Degree between Urban Agglomerations}

(1) Breaking the Segmentation of Urban and Rural Areas and Constructing Two Levels of Economic Growth Pole

The development of Shaanxi requires breaking the partition of urban agglomeration and forming Guanzhong urban agglomeration with the core of Xi'an metropolitan area as its rational spatial structure, clear division of functions and optimized hierarchical structure. Through the integrated development strategy of agglomerative urban agglomeration, the sustainable social, economic and ecological development of the whole region can be realized.

First of all, we should break the urban-rural division and construct the spatial economic and geographical pattern of "one-mind, multi-polarity, urban-rural interaction, opening up both inside and outside" between urban and rural systems. Secondly, we should vigorously develop small and medium-sized cities and towns, realize the gradient transfer of industries and the orderly flow of population, gradually break down the confinement and separation between cities, ease the gap between urban and rural areas, and realize the benign flow of resources, population and technology between urban and rural areas. Finally, Xi'an will be the center and seven surrounding cities will be the two levels of development. Extremely.

(2) Breaking the Urban Segmentation and Forming the Economic Pattern of Urban Integration

We should break the division between cities in urban agglomeration, construct urban agglomeration network, give full play to the "axis" and "belt" arteries of Guanzhong urban agglomeration, and construct a reasonable spatial economic and geographical pattern of urban agglomeration. Guanzhong-Tianshui Economic Zone has broken the urban division between Shaanxi and Gansu provinces, and the construction and layout of Xixian New Area has constructed the economic and geographical spatial pattern of urban integration. In the future, we should take Xi'an Metropolitan Area as the core, strengthen the function of the central city, accelerate and cultivate the urban integrated development zone.

\subsection{Increasing the Mobility and Agglomeration of Urban Population}

(1) Guiding the migration of population and gathering the size of population

The farther away from the economically intensive areas, the less economic integration with the advanced areas. Migration is the most natural way to reduce the distance. Population mobility is the most potential mechanism to achieve market integration in low and high economic density areas. Shortening economic distance, reducing division, further promoting population flow from backward areas to economically intensive areas, and reducing the gap between urban and rural areas and segmentation have become the focus of Shaanxi provincial government policy. The population agglomeration and mobility of Guanzhong urban agglomeration can be seen in Fig 5.1-5.2(Unit: ten thousand). 


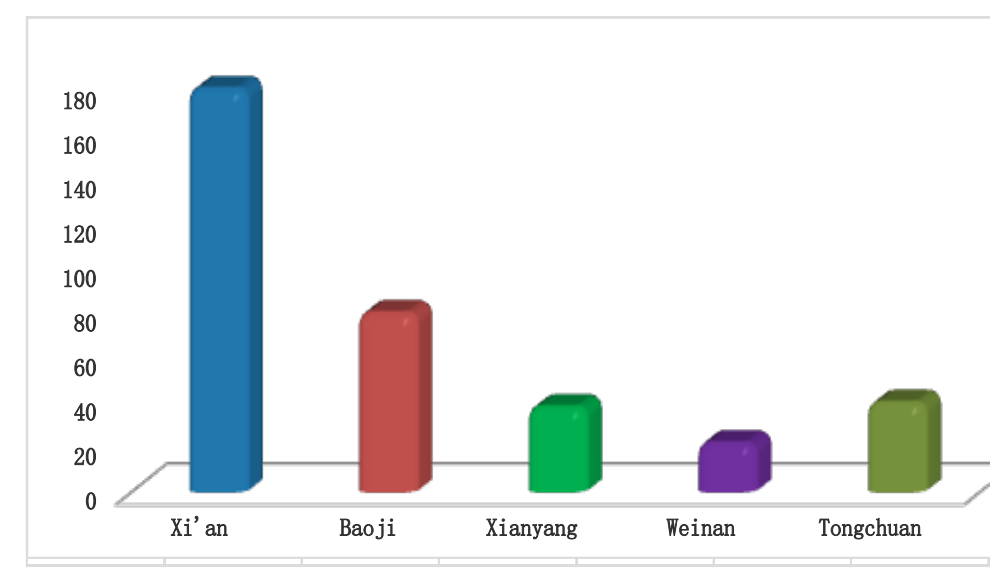

Figure 1 Comparison of population growth in Shaanxi cities from 2000 to 2015

In the past ten years, the new inflow population of Guanzhong urban agglomeration in Shaanxi Province is 1.8 million, which is much higher than that of the surrounding provincial capitals, providing a driving force for the sustained economic growth. In the future, policies should continue to encourage the rational flow of talents and break regional division.

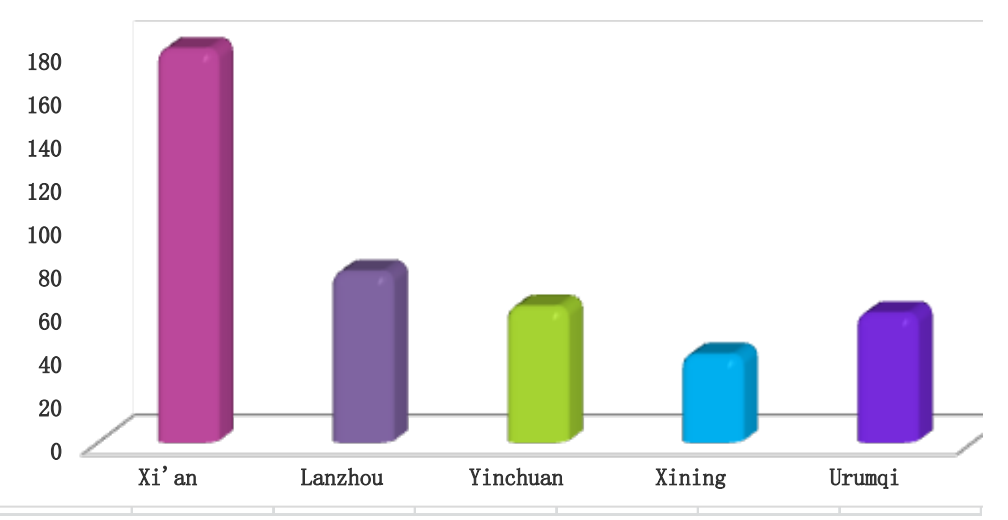

Figure 2 Comparison of urban population growth in peripheral provincial capitals of Shaanxi from 2000 to 2015

(2) Guiding population reflux in resource-exhausted cities

The rapid expansion of Tongchuan's resource industry has resulted in the consecutive shrinkage of traditional manufacturing, service and agriculture, which has restricted the overall economic growth and formed a typical "resource curse" phenomenon. Similar resource-exhausted cities must rely on scientific and technological progress, investment in education, the development of superior manufacturing and agriculture, get out of the trap of relying on resource growth, guide the construction of returning home by policies, and restore the prosperity of the past.

\subsection{Shaanxi Free Trade Zone Layout Helps Realize Industrial Agglomeration Effect}

Shaanxi is located in the core area of the national FTA network, with five special customs regulatory areas, including land ports, comprehensive bonded area, high-tech comprehensive bonded area and export processing area, ranking in the forefront of the West and even the whole country. The four functional areas of Shaanxi FTA, High-tech Zone should attract high-end innovative elements and resources from all over the world to promote international countries.The economic development zone should vigorously develop the advanced manufacturing industry, new financial industry and manufacturing service industry Heights, and build a "one belt and one road" financial 
innovation experimental area and a demonstration area for integration of production and finance. The port area should speed up the construction of international trade platform, international logistics corridor and business innovation; the Chan Ba ecological zone should establish an overseas investment guarantee system, and create a foreign trade commodity exhibition area and an international cultural exchange demonstration area for the countries along the "one belt and one road".

Shaanxi Free Trade Zone, by improving its openness to the outside world economy, plays the synergistic role of Shaanxi Energy, Science and Technology, Education, Culture and Modern Service Trade Innovation Demonstration Park, and reconstructs the economic geography pattern and forms agglomeration in five dominant industries, namely, financial services, trade services, professional services, cultural services and social services. The effect and scale effect will greatly stimulate local economic growth and enhance regional competitiveness.

Shaanxi Guanzhong Urban Agglomeration is the most active, prosperous and technologically advanced area in Northwest China. By reconstructing Shaanxi's economic geography and exploring its new space and pattern of development, it will help Shaanxi realize the transformation of its economic structure, promote the rise of Shaanxi's economy and open a new chapter for the promotion of economic construction in the western region.

\section{References}

[1] Statistical Yearbook of Shaanxi Province in 2015, 2016 and 2017

[2] World Bank Development Report 2009 - Reshaping World Economic Geography [M] Tsinghua University Press, 2008

[3] Reshaping the Economic Geography of Industry in Chengdu, [N] Chengdu Daily, 2017.8.15

[4] Rebuilding Economic Geography and Expanding Development Space, [N] Sichuan Daily, 2018.12.11

[5] Shaanxi Housing and Urban-Rural Construction Department. Research and Practice of New Urbanization Development in Shaanxi Province. [M] China Construction Industry Press, 2016

[6] Lin Ling. Reshaping Economic Geography - World Experience and Choice. [J] Open Report, 2014 